\title{
Homotopy invariance of twisted higher signatures on manifolds with boundary
}

\author{
Bulletin de la S. M. F., tome 127, no 2 (1999), p. 307-331 \\ $<$ http://www.numdam.org/item?id=BSMF_1999_127_2_307_0>
}

(C) Bulletin de la S. M. F., 1999, tous droits réservés.

L'accès aux archives de la revue «Bulletin de la S. M. F. » (http: //smf.emath.fr/Publications/Bulletin/Presentation.html) implique l'accord avec les conditions générales d'utilisation (http://www.numdam.org/ conditions). Toute utilisation commerciale ou impression systématique est constitutive d'une infraction pénale. Toute copie ou impression de ce fichier doit contenir la présente mention de copyright.

\section{Numdam}




\title{
HOMOTOPY INVARIANCE OF TWISTED HIGHER SIGNATURES ON MANIFOLDS WITH BOUNDARY
}

\author{
By Éric LEICHTNAM and PAOLO PIAZZA $\left(^{*}\right)$
}

\begin{abstract}
Let $M$ be an oriented compact manifold with boundary. We assume that $\pi_{1}(M)$ is the product of a non-trivial finite group $F$ and of a group $\Gamma$ which is either of polynomial growth or Gromov hyperbolic. We fix a non-trivial representation $\rho: F \rightarrow U(\ell)$ and let $E_{\rho}$ be the associated unitary flat bundle on $M_{\dot{\widetilde{M}}}$ We denote by $\widetilde{M}$ the universal cover of $M$ and we consider the $\Gamma$-Galois covering $\pi: \widetilde{M} / F \rightarrow M$, the lifted flat bundle $\widetilde{E}_{\rho}=\pi^{*}\left(E_{\rho}\right)$ and the associated twisted signature operator. Under the additional assumption that the induced twisted signature operator on the boundary of $\widetilde{M} / F$ is $L^{2}$-invertible, Lott has introduced in [L2] the twisted higher signatures of $M$; our main result, a positive answer to a form of Novikov conjecture on manifolds with boundary, is that these are homotopy invariants of the pair $(M, \partial M)$. The proof depends heavily on the $b-\mathcal{B}^{\infty}$-pseudodifferential calculus developed in [LP1], on the higher APS index theorem of [LP1] (here extended so as to cover Gromov-hyperbolic groups) and on a classical result of Kaminker-Miller, stating the equality of the index classes associated to two homotopy-equivalent hermitian Fredholm complexes.
\end{abstract}

RÉSUMÉ. - INVARIANCE PAR HOMOTOPIE DES HAUTES SIGNATURES “TWISTÉES" SUR DES VARIÉTÉS À BORD. - Soit $M$ une variété compacte orientée à bord. On suppose que $\pi_{1}(M)$ est le produit d'un groupe fini non trivial $F$ et d'un groupe $\Gamma$ qui est soit à croissance polynomiale, soit hyperbolique au sens de Gromov. On se donne une représentation non triviale $\rho: F \rightarrow U(\ell)$ et on considère le fibré plat unitaire associé $E_{\rho}$. On désigne par $\widetilde{M}$ le revêtement universel de $M$ et on considère le revêtement $\Gamma$-galoisien $\pi: \widetilde{M} / F \rightarrow M$, le fibré plat relevé $\widetilde{E}_{\rho}=\pi^{*}\left(E_{\rho}\right)$ et l'opérateur de signature 《twisté» associé. Sous l'hypothèse supplémentaire que l'opérateur de signature 《twisté»》 induit sur le bord de $\widetilde{M} / F$ est $L^{2}$-inversible, Lott a introduit dans [L2] les hautes signatures 《twistées》 de $M$. Notre résultat principal - une réponse positive à une conjecture de type Novikov pour les variétés à bord - est que ce sont des

(*) Texte reçu le 19 novembre 1998, accepté le $1^{\text {er }}$ février 1999.

E. LeichtnAm, École Normale Supérieure, CNRS, DMI, 45 rue d'Ulm, 75230 Paris CEDEx 05. Email: Eric.Leichtnam@ens.fr.

P. PiazzA, Università di Roma "La Sapienza", Istituto "Guido Castelnuovo", P. le A. Moro 2, I-00185 Roma (Italy). Email: piazza@mat.uniroma1.it.

AMS classification: $58 \mathrm{G} 12,46 \mathrm{~L} 87,58 \mathrm{G} 15$.

Keywords: homotopy invariants, higher signatures, signature operator, $L$-class, universal cover, Atiyah-Patodi-Singer higher index theory, higher eta invariants, $b$-pseudodifferential calculus.

BULlETiN DE LA SOCIÉTÉ MATHÉMATIQUE DE FRANCE 0037-9484/1999/307/\$5.00 (C) Société mathématique de France 
invariants d'homotopie de la paire $(M, \partial M)$. La preuve dépend de manière essentielle du $b-\mathcal{B}^{\infty}$-calcul pseudodifférentiel développé dans [LP1], du théorème d'indice supérieur APS de [LP1] (étendu ici au cas des groupes hyperboliques au sens de Gromov) et du résultat classique de Kaminker-Miller énonçant l'égalité des classes d'indices associées à deux complexes hermitiens de Fredholm homotopiquement équivalents.

\section{Contents}

0. Introduction and main results

1. Proof of the main theorem

2. Examples

3. Remarks on the gap case

4. Appendix: The higher APS index formula for finitely presented groups

\section{Introduction and main results}

Let $M$ be an oriented $4 k$-dimensional compact manifold with boundary. We fix a Riemannian metric $g$ on $M$ which is a product near the boundary, we consider the Levi-Civita connection $\nabla^{M}$ and the Hirzebruch $L$-differential form $L\left(M, \nabla^{M}\right)$. Let $\mathrm{D}$ be the signature operator on $M$ and let $\eta \equiv \eta\left(\mathrm{D}_{0}\right)$ be the eta invariant associated to the odd signature operator $\mathrm{D}_{0}$ induced on the boundary $\partial M$; thus, by definition,

$$
\eta\left(\mathrm{D}_{0}\right)=\frac{2}{\sqrt{\pi}} \int_{0}^{+\infty} \operatorname{Tr}\left(\mathrm{D}_{0} \mathrm{e}^{-\left(t \mathrm{D}_{0}\right)^{2}}\right) \mathrm{d} t
$$

Consider, finally, the difference

$$
\int_{M} L\left(M, \nabla^{M}\right)-\frac{1}{2} \eta
$$

This difference is a homotopy invariant of the pair $(M, \partial M)$. By this we mean that if $M^{\prime}$ is another oriented $4 k^{\prime}$-dimensional manifold with boundary, $g^{\prime}$ a Riemannian metric on $M^{\prime}$ which is a product near the boundary and if $f:(M, \partial M) \rightarrow\left(M^{\prime}, \partial M^{\prime}\right)$ is a homotopy equivalence of oriented pairs, then

$$
\int_{M} L\left(M, \nabla^{M}\right)-\frac{1}{2} \eta\left(\mathrm{D}_{0}\right)=\int_{M^{\prime}} L\left(M^{\prime}, \nabla^{M^{\prime}}\right)-\frac{1}{2} \eta\left(\mathrm{D}_{0}^{\prime}\right)
$$

The proof of the homotopy invariance of $(0.2)$ is the consequence of two non-trivial results.

TOME $127-1999-\mathrm{N}^{\circ} 2$ 
- First, the Atiyah-Patodi-Singer index theorem, which equates (0.2) to

$$
\operatorname{ind}\left(\mathrm{D}^{+}, \Pi_{\geq}\right)+\frac{1}{2} \operatorname{dim} \operatorname{Ker}\left(\mathrm{D}_{0}\right)
$$

with $\left(\mathrm{D}^{+}, \Pi_{\geq}\right)$denoting, as usual, the Atiyah-Patodi-Singer boundary value problem.

- Second, the Hodge theorem on $M$, which equates the number

$$
\operatorname{ind}\left(\mathrm{D}^{+}, \Pi_{\geq}\right)+\frac{1}{2} \operatorname{dim} \operatorname{Ker}\left(\mathrm{D}_{0}\right)
$$

with the signature of $M$, an a priori homotopy invariant. It is also possible to twist everything by a flat bundle $E$ of rank $\ell$; in this case the number

$$
\ell \int_{M} L\left(M, \nabla^{M}\right)-\frac{1}{2} \eta_{E}
$$

with $\eta_{E}$ the eta invariant associated to $\left(\mathrm{D}_{E}\right)_{0}$, is a homotopy invariant of $M$, since it is equal to the signature of $M$ with values in $E$ (see [APS2] and $[\mathrm{Lu}])$.

The homotopy invariance of (0.2) extends to manifolds with boundary the well known result about closed manifolds; on a closed manifold $N$ the characteristic number given by the integral over $N$ of $L\left(N, \nabla^{N}\right)$ is a homotopy invariant. If $N$ is simply connected this number is essentially the only homotopy invariant of $N$ expressible in terms of rational characteristic classes. However, if the fundamental group of $N$ is not trivial there are more (potential) homotopy invariants of this kind; these are the higher Novikov signatures of $N$. We recall the definition.

Let $\Gamma$ be the fundamental group of $N$. Let $\nu: N \rightarrow B \Gamma$ be the classifying map associated to the universal cover of $N$. Fix a cohomology class

$$
[c] \in H^{*}(B \Gamma, \mathbb{C}) \equiv H^{*}(\Gamma, \mathbb{C})
$$

and consider the pullback

$$
\nu^{*}[c] \in H^{*}(N, \mathbb{C}) \equiv H_{\mathrm{dR}}^{*}(N, \mathbb{C}) .
$$

Let $\mathcal{L}(N) \in H_{\mathrm{dR}}^{*}(N, \mathbb{C})$ be the L-class:

$$
\mathcal{L}(N) \equiv\left[L\left(N, \nabla^{N}\right)\right]
$$

As $[c]$ varies in $H^{*}(B \Gamma, \mathbb{C})$ the numbers

$$
\int_{N} \mathcal{L}(N) \wedge \nu^{*}[c]
$$

define the higher signatures of $N$. The Novikov conjecture asserts that all these numbers are homotopy invariants of the closed manifold $N$. The conjecture is still open in general although it has been proved for a number of interesting cases and with a variety of different techniques. We refer to $[\mathrm{FRR}]$ for a very nice (and relatively updated) survey. 
Notice that the expression (0.4) is not well defined on a manifold with boundary (the cohomology class $\mathcal{L}(N) \cup \nu^{*}[c]$ appearing in (0.4) is an absolute cohomology class which cannot be paired with the fundamental class $[N])$.

Among the most successfull appoaches to the conjecture is the one that employs higher index theory for the signature operator. This method, due to Lusztig in the commutative case and then to Connes and Moscovici in the noncommutative setting, applies to closed manifolds with a fundamental group $\Gamma$ which is either Gromov-hyperbolic or virtually nilpotent (i.e. of polynomial growth with respect to a word metric). Conceptually the proof of the homotopy invariance of (0.4), as given by Lusztig and Connes-Moscovici in these two cases, follows the two steps sketched at the beginning of this introduction. Namely, one first establishes a higher index theorem, expressing (0.4) in terms of the Chern character of an index class defined by the signature operator, and then proves that such an index class is a homotopy invariant. Since these two steps are fundamental in what follows, we give a few more details. The higher index theorem is obtained as follows; the signature operator defines an index class $\operatorname{Ind}\left(\mathcal{D}^{+}\right)$in $K_{0}\left(C_{r}^{*}(\Gamma)\right)$. The reduced $C^{*}$-algebra of the group should be thought of as the space of continuous functions on a noncommutative space. It is possible, in general, to construct a dense subalgebra

$$
\mathcal{B}_{\Gamma}^{\infty} \equiv \mathcal{B}^{\infty} \quad \text { of } \quad C_{r}^{*}(\Gamma), \quad \mathbb{C} \Gamma \subset \mathcal{B}^{\infty}
$$

playing the role of the $C^{\infty}$-functions, which is dense in $C_{r}^{*}(\Gamma)$ and closed under holomorphic functional calculus (see, for example, the Appendix). If $\Gamma$ is of polynomial growth then $\mathcal{B}^{\infty}$ is nothing but the subalgebra of rapidly decreasing functions on $\Gamma$. Since by assumption the algebra $\mathcal{B}^{\infty}$ is dense in $C_{r}^{*}(\Gamma)$ and closed under holomorphic functional calculus, we have $K_{0}\left(C_{r}^{*}(\Gamma) \cong K_{0}\left(\mathcal{B}^{\infty}\right)\right.$; thus $\operatorname{Ind}(\mathcal{D}) \in K_{0}\left(\mathcal{B}^{\infty}\right)$.

We can take the Chern character of this index class, with values in Karoubi's non-commutative topological de Rham homology $\bar{H}_{*}\left(\mathcal{B}^{\infty}\right)$, see [Ka]. Recall that Karoubi's (topological) non-commutative de Rham homology always pairs with (topological) cyclic cohomology. Given an element $[c] \in H^{*}(\Gamma, \mathbb{C})$ we can construct a cyclic cocycle $\tau_{c}$ and take its class in $H C^{*}(\mathbb{C} \Gamma)$. If, as we are assuming, the group is virtually nilpotent or Gromov hyperbolic, then there exist a cocycle representative in $[c]$, still denoted by $c$, such that the cyclic cocycle $\tau_{c}$ extends from $Z C^{*}(\mathbb{C} \Gamma)$ to $Z C^{*}\left(\mathcal{B}^{\infty}\right)$. It is precisely at this point that one uses the assumptions on the group $\Gamma$. We can pair the corresponding cyclic cohomology class in $H C^{*}\left(\mathcal{B}^{\infty}\right)$ with the homology class

$$
\operatorname{Ch}\left(\operatorname{Ind}\left(\mathcal{D}^{+}\right)\right) \in \bar{H}_{*}\left(\mathcal{B}^{\infty}\right)
$$

TOME $127-1999-\mathrm{N}^{\circ} 2$ 
thus obtaining a number:

$$
\left\langle\operatorname{Ch}\left(\operatorname{Ind}\left(\mathcal{D}^{+}\right)\right), \tau_{c}\right\rangle
$$

The higher index theorem of Connes-Moscovici states that

$$
\left\langle\operatorname{Ch}\left(\operatorname{Ind}\left(\mathcal{D}^{+}\right)\right), \tau_{c}\right\rangle=\int_{N} \mathcal{L}(N) \wedge \nu^{*}[c]
$$

This result gives an analytic interpretation of the higher signatures. The second step in the proof of (this case of) the Novikov conjecture, namely the proof of the homotopy invariance of the index class

$$
\operatorname{Ind}\left(\mathcal{D}^{+}\right) \in K_{0}\left(C_{r}^{*}(\Gamma)\right)
$$

can either be carried out directly, as in $[\mathrm{KM}][\mathrm{HS}]$, or by showing that this index class is equal to an a priori homotopy invariant, the Mishenko symmetric signature of $M$.

As a last piece of background material we mention Lott's heat kernel proof of the Connes-Moscovici higher index theorem. First recall that Karoubi's definition of noncommutative de Rham homology is based on a space of noncommutative "smooth" differential forms $\widehat{\widehat{\Omega}}\left(\mathcal{B}^{\infty}\right)$. Lott's heat kernel proof of the Connes-Moscovici result implies the existence of an explicit representative for the non-commutative de Rham class $\operatorname{Ch}\left(\operatorname{Ind}\left(\mathcal{D}^{+}\right)\right)$:

$$
\operatorname{Ch}\left(\operatorname{Ind}\left(\mathcal{D}^{+}\right)\right)=\left[\int_{N} L\left(N, \nabla^{N}\right) \wedge \omega\right]
$$

with $\omega \in \Omega^{*}(N) \otimes \overline{\widehat{\Omega}}\left(\mathcal{B}^{\infty}\right)$ a closed explicit bi-form. We shall call $\omega$ the Lott bi-form of the Galois covering $\Gamma \rightarrow \widetilde{N} \rightarrow N$. As a last important step Lott also proves that, if $\Gamma$ is either of polynomial growth or Gromov hyperbolic, then

$$
\left\langle\left[\int_{N} L\left(N, \nabla^{N}\right) \wedge \omega\right], \tau_{c}\right\rangle=\int_{N} \mathcal{L}(N) \wedge \nu^{*}[c]
$$

thus reobtaining the higher index theorem of Connes-Moscovici. The heat kernel approach developed by Lott also allows for the introduction of a higher eta invariant $\widetilde{\eta}$, a noncommutative differential form generalizing (0.1) (see [L2, p. 214] for the polynomial growth case and both [L3] and the Appendix for the general case). 
The main aim of this article is to give a (partial) answer to the following rather fundamental

Question. - If $M$ is a manifold with boundary, can one define higher signatures, generalizing (0.4), and prove their homotopy invariance?

That this is not a trivial extension of the closed case already appears from our discussion above, leading to the homotopy invariance of (0.2) on a manifold with boundary. The question was first addressed in [L2] where, as already remarked, a candidate for the boundary correction term (the higher eta invariant) was introduced for operators $\widetilde{D}$ of Dirac-type which are $L^{2}$-invertible. Because of this last requirement the higher signatures defined in [L2, p. 228]) are twisted. It is only by twisting the signature operator that we can reasonably assume an invertible signature laplacian on the boundary; an invertibility assumption on the untwisted boundary signature laplacian would be too strong, see [L4].

We shall make two hypothesis. First we assume that

$$
\left(H_{1}\right) \quad\left\{\begin{array}{l}
\pi_{1}(M)=F \times \Gamma \text { with } F \text { a non-trivial finite group and } \\
\Gamma \text { either of polynomial growth or Gromov hyperbolic. }
\end{array}\right.
$$

In order to state our second hypothesis we need to introduce some notation. We fix $\rho: F \rightarrow U(\ell)$, a non-trivial representation of $F$. We let $E_{\rho}$ be the corresponding flat unitary bundle on $M$ and $\mathrm{D}_{\rho}$ the associated twisted signature operator. Consider the $\Gamma$-Galois covering $\pi:(\widetilde{M} / F) \rightarrow M$, with $\widetilde{M}$ the universal cover of $M$, and the twisting flat bundle $\widetilde{E}_{\rho}=\pi^{*}\left(E_{\rho}\right)$; let $\widetilde{\mathrm{D}}_{\rho}^{\Gamma}$ the associated twisted signature operator and $\left(\widetilde{\mathrm{D}}_{\rho}^{\Gamma}\right)_{0}$ the induced boundary signature operator on $\partial(\widetilde{M} / F)$. Our second assumption reads:

$\left(H_{2}\right) \quad$ The boundary operator $\left(\widetilde{\mathrm{D}}_{\rho}^{\Gamma}\right)_{0}$ is $L^{2}$-invertible.

Notice that the two assumptions $\left(H_{1}\right)$ and $\left(H_{2}\right)$ are homotopy invariant conditions of the pair $(M, \partial M)$. Because of assumption $\left(H_{2}\right)$ we can define, following [L2], [L3] and the Appendix, a twisted higher eta invariant, $\widetilde{\eta}_{\rho}$. Finally let

$$
\omega \in \Omega^{*}(M) \otimes \widehat{\Omega}_{*}\left(\mathcal{B}^{\infty}\right)
$$

be the Lott's biform (see [LP1, p. 95]) associated to the covering

$$
\Gamma \longrightarrow \widetilde{M} / F \longrightarrow M \text {; }
$$

here the algebra $\mathcal{B}^{\infty}$ stands for the algebra of rapidly decreasing functions on $\Gamma$ in the virtually nilpotent case and for the Connes-Moscovici algebra in the hyperbolic case (see $[\mathrm{CM}]$ ). We can now state the main result of this paper:

$$
\text { Tоме } 127-1999-\mathrm{N}^{\circ} 2
$$


THEOREM 0.1. - Let $M$ be a compact oriented manifold with boundary satisfying the two assumptions $\left(H_{1}\right),\left(H_{2}\right)$ above. Let $[c] \in H^{*}(\Gamma, \mathbb{C})$ and let $\tau_{c} \in H C^{*}(\mathbb{C} \Gamma)$ be the cyclic cohomology class associated to the corresponding (extendable) cyclic cocycle. Then the complex numbers

$$
\left\langle\ell \int_{M} L\left(M, \nabla^{M}\right) \wedge \omega-\frac{1}{2} \widetilde{\eta}_{\rho}, \tau_{c}\right\rangle
$$

are homotopy invariants of the pair $(M, \partial M)$.

In simple words our main theorem says the following: if the manifold has a boundary the integral appearing in (0.6) does not define a closed non-commutative differential form. In particular it does not pair with the cyclic cocycles $\tau_{c}$ and cannot produce homotopy invariants. Theorem 0.1 identifies the boundary correction term we have to subtract in order to obtain homotopy invariants, at least in some special cases. The proof, rigorously presented in the next section, is based on an extension to hyperbolic groups of the higher Atiyah-Patodi-Singer index formula of [LP1] (see the Appendix), the $b-\mathcal{B}^{\infty}$-Mishenko-Fomenko calculus (developed in [LP1]) and on a result of Kaminker-Miller [KM] stating the equality of the index class associated to two hermitian Fredholm complexes which are chain homotopy equivalent.

The results of this paper were announced in [LP3]. The paper has been circulating as Preprint IHES/M/97/89.

Acknowledgements. - We would like to thank Jean-Michel Bismut, John Lott and Richard Melrose for their interest in this work and for several helpful and stimulating discussions. Part of this work was done while the first author was visiting Università di Roma "La Sapienza" and while the second author was visiting École Normale Supérieure and Université de Paris Sud. Financial support for these visits was provided by a CNR-CNRS cooperation project and by a Marie Curie Research Training Grant of the European Community. We are very grateful to all these institutions for their hospitality and/or financial support.

\section{Proof of the main theorem}

We are assuming that $\pi_{1}(M)=F \times \Gamma$, with $F$ finite and $\Gamma$ either of polynomial growth or Gromov hyperbolic. Let $\pi_{1}(M) \rightarrow \widetilde{M} \rightarrow M$ the universal cover of $M$. We then obtain two additional Galois coverings:

$$
F \longrightarrow(\widetilde{M} / \Gamma) \longrightarrow M, \quad \Gamma \longrightarrow(\widetilde{M} / F) \longrightarrow M
$$


We shall be mainly concerned with the second one; we denote the noncompact covering space $(\widetilde{M} / F)$ by $\widetilde{M}^{\Gamma}$ so as to make precise the fact that this is a $\Gamma$-Galois covering.

Let $\rho: F \rightarrow U(\ell)$ be the non-trivial representation of hypothesis $\left(H_{2}\right)$ and let $E_{\rho} \rightarrow M$ be the corresponding flat unitary bundle. We also have a flat unitary bundle $\widetilde{E}_{\rho}$ over $\widetilde{M}^{\Gamma}$ and it is clear that this is simply the pull-back of $E_{\rho}$ under the obvious covering map $\pi: \widetilde{M}^{\Gamma} \rightarrow M$.

We shall now define the various signature operators that will be needed in the proof of the theorem. By $\mathcal{B}^{\infty}$ we denote either the subalgebra of $C_{r}^{*}(\Gamma)$ of rapidly decreasing functions, in the case $\Gamma$ of polynomial growth, or the Connes-Moscovici algebra, in the case $\Gamma$ Gromov hyperbolic. Recall that $K_{0}\left(C_{r}^{*}(\Gamma)\right) \cong K_{0}\left(\mathcal{B}^{\infty}\right)$. We shall consider the following $C_{r}^{*}(\Gamma)$ and $\mathcal{B}^{\infty}$-flat bundles over $M$ :

$$
\mathcal{V}_{\rho}^{\Gamma}=\left(\widetilde{M}^{\Gamma} \times_{\Gamma} C_{r}^{*}(\Gamma)\right) \otimes_{\mathbb{C}} E_{\rho}, \quad \mathcal{V}_{\rho}^{\Gamma}(\infty)=\left(\widetilde{M}^{\Gamma} \times_{\Gamma} \mathcal{B}^{\infty}\right) \otimes_{\mathbb{C}} E_{\rho}
$$

We shall denote by $\mathcal{D}_{\rho}^{\Gamma}$ the twisted $b$-signature operator acting on the $C_{r}^{*}(\Gamma)$-Hilbert modules $\mathcal{H}_{b}^{k}\left(M, \mathcal{V}_{\rho}^{\Gamma} \otimes{ }^{b} \Lambda^{*} M\right)$, where $k \in \mathbb{N}$, or on the space $\mathcal{H}_{b}^{\infty}\left(M, \mathcal{V}_{\rho}^{\Gamma}(\infty) \otimes{ }^{b} \Lambda^{*} M\right)$ as defined in the $b$-Mishenko-Fomenko calculus of [LP1]. The corresponding boundary operator will be denoted by $\left(\mathcal{D}_{\rho}^{\Gamma}\right)_{0}$; it acts on $C^{\infty}\left(\partial M, \mathcal{V}_{\rho}^{\Gamma} \mid \partial M \otimes \Lambda^{*}(\partial M)\right)$.

Lott has established a relationship between the lifted signature operator $\widetilde{\mathrm{D}}_{\rho}^{\Gamma}$ on the covering $\widetilde{M}^{\Gamma}$, acting on the sections of $\pi^{*}\left(E_{\rho} \otimes{ }^{b} \Lambda^{*} M\right)$, and the operator $\mathcal{D}_{\rho}^{\Gamma}$. For simplicity we describe it only when $\Gamma$ is virtually nilpotent. Under this latter assumption, Lott has established a correspondence between $\mathcal{B}^{\infty}$-sections on the base and rapidly decreasing sections on the $\Gamma$-covering $\pi: \widetilde{M}^{\Gamma} \rightarrow M$ (see [L2, p. 221]). For any section $u$ in the Schwartz space $\mathcal{S}_{b}\left(\widetilde{M}^{\Gamma}, \pi^{*}\left(E_{\rho} \otimes{ }^{b} \Lambda^{*} M\right)\right)$ the element

$$
\sum_{\gamma \in \Gamma} R_{\gamma}^{*}(u) \gamma
$$

belongs to $\mathcal{H}_{b}^{\infty}\left(M, \mathcal{V}_{\rho}^{\Gamma}(\infty) \otimes{ }^{b} \Lambda^{*} M\right)$, with $\mathcal{H}_{b}^{\infty}=\bigcap \mathcal{H}_{b}^{k}$, and conversely. The lifted signature operator $\widetilde{\mathrm{D}}_{\rho}^{\Gamma}$ acting on $\mathcal{S}_{b}\left(\widetilde{M}^{\Gamma}, \pi^{*}\left(E_{\rho} \otimes{ }^{b} \Lambda^{*} M\right)\right)$ then satisfies

$$
\mathcal{D}_{\rho}^{\Gamma}\left[\sum_{\gamma \in \Gamma} R_{\gamma}^{*}(u) \gamma\right]=\sum_{\gamma \in \Gamma} R_{\gamma}^{*}\left(\widetilde{\mathrm{D}}_{\rho}^{\Gamma}(u)\right) \gamma .
$$

Now we go back to the general case, assuming $\Gamma$ to be either hyperbolic or virtually nilpotent. The corresponding signature operator on $\partial\left(\widetilde{M}^{\Gamma}\right)$ will be denoted, as usual, by $\left(\widetilde{\mathrm{D}}_{\rho}^{\Gamma}\right)_{0}$.

$$
\text { TOME } 127-1999-\mathrm{N}^{\circ} 2
$$


Proposition 1.1. - The boundary operator $\left(\mathcal{D}_{\rho}^{\Gamma}\right)_{0}$ is invertible both in the $C_{r}^{*}(\Gamma)$ - and in the $\mathcal{B}^{\infty}$-calculus. Moreover the graded signature operator $\left(\mathcal{D}_{\rho}^{\Gamma}\right)^{+}$on the manifold $M$ is $C_{r}^{*}(\Gamma)$-Fredholm.

Proof. - By assumption $\left(H_{1}\right)$ the boundary operator $\left(\widetilde{\mathrm{D}}_{\rho}^{\Gamma}\right)_{0}$ is $L^{2}$ invertible. Thus we can find an $\varepsilon>0$ and $\chi \in C^{\infty}(\mathbb{R}, \mathbb{R})$ such that $\chi(x)=0$ (respectively $=1 / x$ ) for $|x|<\varepsilon$ (resp. $>2 \varepsilon$ ) in such a way that the inverse of $\left(\widetilde{\mathrm{D}}_{\rho}^{\Gamma}\right)_{0}$ is given by $\chi\left(\left(\widetilde{\mathrm{D}}_{\rho}^{\Gamma}\right)_{0}\right)$. Using Lott's correspondence one checks immediatly that the operator $\chi\left(\left(\mathcal{D}_{\rho}^{\Gamma}\right)_{0}\right)$ provides an inverse for $\left(\mathcal{D}_{\rho}^{\Gamma}\right)_{0}$. The fact that $\left(\mathcal{D}_{\rho}^{\Gamma}\right)^{+}$is $C_{r}^{*}(\Gamma)$-Fredholm as an operator $\mathcal{H}_{b}^{1} \rightarrow L_{b}^{2}$ is a consequence of the results in [LP1, p. 15].

Since $E_{\rho}$ is a flat unitary vector bundle, $\mathcal{V}_{\rho}^{\Gamma}$ is endowed with a natural $C_{r}^{*}(\Gamma)$-Hermitian product denoted $(;)$ which we will take to be antilinear on the left. Let

$$
\operatorname{dim}(M)=2 m \text {. }
$$

Then given $x \in M$ and $k \in\{-m,-m+1, \ldots, m-1, m\}$ we set

$$
\langle\xi \otimes \alpha ; \eta \otimes \beta\rangle_{x}=\epsilon(\bar{\alpha} \wedge \beta)(\xi ; \eta)
$$

for any $\xi, \eta \in \mathcal{V}_{\rho}^{\Gamma}, \alpha \in{ }^{b} \Lambda_{x}^{m-k}(M), \beta \in{ }^{b} \Lambda_{x}^{m+k}(M)$ and with

$$
\epsilon= \begin{cases}1 & \text { if }(m-k) \text { is even } \\ \sqrt{-1} & \text { if }(m-k) \text { is odd }\end{cases}
$$

Moreover we set

$$
C^{k} \equiv \mathcal{H}_{b}^{k}\left(M, \mathcal{V}_{\rho}^{\Gamma} \otimes_{\mathbb{C}}{ }^{b} \Lambda^{m-k}(M)\right)
$$

For any $k \in\{-m, \ldots, m\}$ we get a map

$$
\begin{gathered}
C^{k} \times C^{-k} \longrightarrow C_{r}^{*}(\Gamma), \\
(u, v) \longmapsto \int_{M}\langle u ; v\rangle_{x} \equiv\langle u ; v\rangle
\end{gathered}
$$

which is well defined. Indeed $C^{k}$ is the completion of

$$
C_{c}^{\infty}\left(M \backslash \partial M, \mathcal{V}_{\rho}^{\Gamma} \otimes{ }^{b} \Lambda^{*}(M)\right)
$$

with respect to the $C_{r}^{*}(\Gamma)$-Hermitian product

$$
\langle\langle u ; v\rangle\rangle_{k}=\left\langle\left(\mathrm{id}+\Delta_{\rho}\right)^{k} u ; v\right\rangle
$$

with

$$
\Delta_{\rho}=\mathrm{d}_{\rho}^{*} \mathrm{~d}_{\rho}+\mathrm{d}_{\rho} \mathrm{d}_{\rho}^{*}
$$

equal to the $b$-Hodge Laplacian associated to the twisted exterior derivative $\mathrm{d}_{\rho}$. 
We shall now make use of the notion of graded Hermitian Fredholm complex as given in [KM].

Proposition 1.2. - The bounded complex

$$
\cdots \longrightarrow C^{k} \rightarrow C^{k+1} \longrightarrow \cdots
$$

endowed with the differential $\tilde{\mathrm{d}}_{\rho}$ on $C^{k}$

$$
\widetilde{\mathrm{d}}_{\rho}= \begin{cases}\mathrm{d}_{\rho} & i m-k=2 \ell, \\ -\sqrt{-1} \mathrm{~d}_{\rho} & \text { if } m-k=2 \ell+1,\end{cases}
$$

and with the maps $C^{k} \times C^{-k} \rightarrow C_{r}^{*}(\Gamma),(u, v) \mapsto\langle u ; v\rangle$, is a graded Hermitian Fredholm complex.

Proof. - In order to prove that this bounded cochain complex is Hermitian we need to check the following four conditions: for all $(u, v)$ in $C^{k} \times C^{-k}$ and for all $a$ in $C_{r}^{*}(\Gamma)$,

(i) $\langle u ; v a\rangle=\langle u ; v\rangle a$;

(ii) $\langle u ; v\rangle^{*}=\langle v ; u\rangle$;

(iii) $\left\langle\tilde{d}_{\rho}(u) ; w\right\rangle=\left\langle u ; \tilde{d}_{\rho}(w)\right\rangle$ for all $u \in C^{k}$ and all $w \in C^{-k+1}$;

(iv) let $C^{\prime}$ be the dual complex:

$$
\left(C^{\prime}\right)^{k}=\text { dual of } C^{-k}, \quad \mathrm{~d}^{\prime}=\text { transpose of } \widetilde{\mathrm{d}}_{\rho} .
$$

Then $\langle;\rangle: C \rightarrow C^{\prime}$ is a chain homotopy equivalence of complexes.

We leave (i)-(iv) to the reader.

In order to prove that the Hermitian complex $\left(C^{*}, \widetilde{\mathrm{d}}_{\rho}\right)$ is Fredholm we have to construct a map $h: C^{*} \rightarrow C^{*}$ of degree -1 such that

$$
h \tilde{\mathrm{d}}_{\rho}+\tilde{\mathrm{d}}_{\rho} h-\mathrm{id}
$$

is $C_{r}^{*}(\Gamma)$-compact. Since the boundary signature operator $\left(\mathcal{D}_{\rho}^{\Gamma}\right)_{0}$ is invertible we can adapt the arguments given in [Me, p. 175] and find $A$ in $\Psi_{b}^{-\infty, \varepsilon, \varepsilon}\left(M, \mathcal{V}_{\rho}^{\Gamma} \otimes{ }^{b} \Lambda^{*} M\right)$ such that the indicial family of

$$
\left(\tilde{\mathrm{d}}_{\rho} \tilde{\mathrm{d}}_{\rho}^{*}+\tilde{\mathrm{d}}_{\rho}^{*} \tilde{\mathrm{d}}_{\rho}\right)\left[\left(\mathrm{id}+\Delta_{\rho}\right)^{-1}+A\right]
$$

is the identity. Moreover one checks that the indicial family of $\tilde{\mathrm{d}}_{\rho}$ commutes with that of $\left(\mathrm{id}+\Delta_{\rho}\right)^{-1}+A$. Thus

$$
h:=\tilde{\mathrm{d}}_{\rho}^{*}\left[\left(\mathrm{id}+\Delta_{\rho}\right)^{-1}+A\right]
$$

is such that $h \tilde{\mathrm{d}}_{\rho}+\tilde{\mathrm{d}}_{\rho} h$-id is $C_{r}^{*}(\Gamma)$-compact. The proposition is proved.

TOME $127-1999-\mathrm{N}^{\circ} 2$ 
Using Proposition 1.1 and the classical result of $[\mathrm{MF}]$ we can define an index class $\operatorname{Ind}\left(\mathcal{D}_{\rho}^{\Gamma}\right)^{+} \in K_{0}\left(C_{r}^{*}(\Gamma)\right)$.

Proposition 1.3. - The index class $\operatorname{Ind}\left(\mathcal{D}_{\rho}^{\Gamma}\right)^{+}$is a homotopy invariant of the oriented pair $(M, \partial M)$.

Proof. - We consider a smooth triangulation $\mathcal{T}(M)$ of $M$ inducing a smooth triangulation $\mathcal{T}(\partial M)$ of $\partial M$. We follow [Lu, p. 247] and [KM, p. 123]. Let

$$
\mathcal{C}_{\text {rel }, k}, \quad 0 \leq k \leq 2 m
$$

the vector space of $\mathbb{C}$-valued relative $k$-simplicial chains associated to $\mathcal{T}(M)$. We denote by $\boldsymbol{e}_{k}$ the canonical basis of $\mathcal{C}_{\text {rel }, k}$. We also consider the dual space of relative $k$-simplicial cochains $\mathcal{C}_{\text {rel }}^{k}$ with the dual basis $\boldsymbol{e}^{k}$. We now consider $C_{\text {rel }}^{j}\left(M ; \mathcal{V}_{\rho}^{\Gamma}\right),-m \leq j \leq m$, the space of $(j+m)$-relative cochains with values in the flat bundle $\mathcal{V}_{\rho}^{\Gamma}$. We shall use the standard coboundary operator

$$
\mathrm{d}: C_{\mathrm{rel}}^{j}\left(M ; \mathcal{V}_{\rho}^{\Gamma}\right) \longrightarrow C_{\mathrm{rel}}^{j+1}\left(M ; \mathcal{V}_{\rho}^{\Gamma}\right)
$$

By using the $C_{r}^{*}(\Gamma)$-Hermitian scalar product of $\mathcal{V}_{\rho}^{\Gamma}$ we shall now define a pairing

$$
\langle;\rangle: C_{\text {rel }}^{j}\left(M ; \mathcal{V}_{\rho}^{\Gamma}\right) \times C_{\text {rel }}^{-j}\left(M ; \mathcal{V}_{\rho}^{\Gamma}\right) \longrightarrow C_{r}^{*}(\Gamma) .
$$

First we fix a chain representative $\mu$ of $[M] \in H_{2 m}(M, \partial M)$; next we observe that each element $x$ in $C_{\text {rel }}^{j}\left(M ; \mathcal{V}_{\rho}^{\Gamma}\right)$ can be identified with a sum of elements like $\xi \otimes \alpha$ with $\alpha$ a $(m+j)$-relative-cochain in $M$ belonging to the dual basis $e^{m+j}$ and $\xi \in C_{r}^{*}(\Gamma) \otimes_{\mathbb{C}} \mathbb{C}^{\ell}$ with $\ell=\operatorname{rank} E_{\rho}$. Let $y=\eta \otimes \beta$ be an element in $C_{\text {rel }}^{-j}\left(M ; \mathcal{V}_{\rho}^{\Gamma}\right)$, thus with $\beta$ a $(m-j)$-relative-cochain in $\boldsymbol{e}^{m-j}$; we set

$$
\langle x ; y\rangle=\frac{1}{2} \epsilon\left(\bar{\alpha} \cup \beta+(-1)^{m^{2}-j^{2}} \beta \cup \bar{\alpha}\right)(\mu)(\xi ; \eta)
$$

with

$$
\epsilon= \begin{cases}1 & \text { if }(m+j) \text { is even, } \\ \sqrt{-1} & \text { if }(m+j) \text { is odd }\end{cases}
$$

This symmetrization is necessary (see $[\mathrm{Lu}]$ ) since at the cochain level $\bar{\alpha} \cup \beta \neq(-1)^{m^{2}-j^{2}} \beta \cup \bar{\alpha}$ in general. Lastly we define

$$
\tilde{\mathrm{d}}: C_{\mathrm{rel}}^{j}\left(M ; \mathcal{V}_{\rho}^{\Gamma}\right) \longrightarrow C_{\text {rel }}^{j+1}\left(M, \mathcal{V}_{\rho}^{\Gamma}\right)
$$

by

$$
\tilde{\mathrm{d}}= \begin{cases}\mathrm{d} & \text { if }(m+j) \text { is even } \\ -\sqrt{-1} \mathrm{~d} & \text { if }(m+j) \text { is odd }\end{cases}
$$

Of course $\tilde{\mathrm{d}}^{2}=0$. 
The following lemma gathers several properties of the complex just defined. Notice that the above triangulation $\mathcal{T}(\partial M)$ allows to define an analogous complex $\left(C^{*}\left(\partial M ; \mathcal{V}_{\rho}^{\Gamma} \mid \partial M\right), \tilde{\mathrm{d}}\right)$.

Lemma 1.4. - The complex $C_{\text {rel }}^{*}\left(M ; \mathcal{V}_{\rho}^{\Gamma}\right)$ is finitely generated and Hermitian. Its signature $\sigma\left(C_{\mathrm{rel}}^{*}\left(M ; \mathcal{V}_{\rho}^{\Gamma}\right)\right)$ belongs to $K_{0}\left(C_{r}^{*}(\Gamma)\right)$ and is an homotopy invariant of the pair $(M, \partial M)$, the non-trivial representation $\rho$ being fixed.

Proof. - Because of the above symmetrization one easily checks that the properties (i), (ii), (iii) (see the proof of Proposition 1.2) are satisfied.

Let us prove (iv): since the boundary signature operator $\left(\mathcal{D}_{\rho}^{\Gamma}\right)_{0}$ is invertible, we can use Hodge and de Rham-type theorems with coefficients in $\mathcal{V}_{\rho \mid \partial M}^{\Gamma}$ in order to show that $C^{*}\left(\partial M ; \mathcal{V}_{\rho \mid \partial M}^{\Gamma}\right)$ is acyclic in all degrees. Similarly the de Rham cohomology groups $H_{\mathrm{dR}}^{*}\left(\partial M ; \mathcal{V}_{\rho}^{\Gamma} \mid \partial M\right)$ are all trivial. Thus, the absolute and relative de Rham cohomology groups of $M$ with coefficients in $\mathcal{V}_{\rho}^{\Gamma}$ both coincide with the cohomology groups of $C_{\text {rel }}^{*}\left(M ; \mathcal{V}_{\rho}^{\Gamma}\right)$. We can now use Poincaré duality to check that $C_{\text {rel }}^{*}\left(M ; \mathcal{V}_{\rho}^{\Gamma}\right)$ satisfies the property (iv) defining an Hermitian complex. Thus $\left(C_{\text {rel }}^{*}\left(M ; \mathcal{V}_{\rho}^{\Gamma}\right), \tilde{\mathrm{d}}_{\rho},\langle;\rangle\right)$ is indeed a finitely generated Hermitian complex. (For the definition of the signature $\sigma\left(C_{\text {rel }}^{*}\left(M ; \mathcal{V}_{\rho}^{\Gamma}\right)\right)$ see $[\mathrm{KM}, \mathrm{p} .120]$.) The homotopy invariance of $\sigma\left(C_{\mathrm{rel}}^{*}\left(M ; \mathcal{V}_{\rho}^{\Gamma}\right)\right)$ follows at once from the main result of [KM, Thm 4.1], since the homotopy type of the Hermitian complex $C_{\text {rel }}^{*}\left(M, \mathcal{V}_{\rho}^{\Gamma}\right)$ clearly depends only on the homotopy type of $(M, \partial M)$. Lemma 1.4 is proved.

We now go back to the proof of Proposition 1.3. We recall (see $[\mathrm{KM}])$ that the signature $\sigma\left(C^{*}\right)$ of the Fredholm Hermitian complex $C^{*}$ of (1.1) is precisely equal to $\operatorname{Ind}\left(\mathcal{D}_{\rho}^{\Gamma}\right)^{+} \in K_{0}\left(C_{r}^{*}(\Gamma)\right)$. This means that Lemma 1.4, the next lemma and Theorem 4.1 of $[\mathrm{KM}]$ immediatly imply Proposition 1.3

LEMMA 1.5. - There is a natural chain homotopy equivalence of Hermitian complexes $f: C_{\mathrm{rel}}^{*}\left(M ; \mathcal{V}_{\rho}^{\Gamma}\right) \rightarrow C^{*}$ with $C^{*}$ defined by (1.1).

Proof. - As in $[\mathrm{KM}]$ we consider the following maps of complexes:

$$
w: C_{\mathrm{rel}}^{*}\left(M ; \mathcal{V}_{\rho}^{\Gamma}\right) \rightarrow \Omega_{\mathrm{rel}}^{*}\left(M ; \mathcal{V}_{\rho}^{\Gamma}\right), \quad j: \Omega_{\mathrm{rel}}^{*}\left(M ; \mathcal{V}_{\rho}^{\Gamma}\right) \rightarrow C^{*}
$$

where $\Omega_{\text {rel }}^{*}\left(M ; \mathcal{V}_{\rho}^{\Gamma}\right)$ is the complex of smooth relative differential forms with coefficients in $\mathcal{V}_{\rho}^{\Gamma}, w$ is the Whitney map (see [Do, p. 162]); $j$ is the inclusion map. Since the boundary operator $\left(\mathcal{D}_{\rho}^{\Gamma}\right)_{0}$ is invertible, we observe that the complex $C^{*}$ is a resolution of the space of flat sections of $\mathcal{V}_{\rho}^{\Gamma}$

TOME $127-1999-\mathrm{N}^{\circ} 2$ 
over $M$. We set $f=j \circ w$. Using appropriate Poincaré lemmas for each of the three complexes above, together with de Rham-type theorems, one checks that $f$ induces an isomorphism in cohomology. Moreover, one sees easily that $f$ satisfies the requirement of Definition 2.2 of [KM]. Thus $f$ is a chain homotopy equivalence of Hermitian complexes and Lemma 1.5 is proved.

Having established Proposition 1.3, i.e. the homotopy invariance of the index class of the signature operator, we can now apply our main result in [LP1, Section 14] together with its extension to the hyperbolic case given in the Appendix. Thus we take a "smooth" representative of $\operatorname{Ind}\left(\mathcal{D}_{\rho}^{\Gamma}\right)^{+}$ in $K_{0}\left(\mathcal{B}^{\infty}\right)$ and apply the main formula in [LP1] to obtain:

$$
\left\langle\ell \int_{M} L\left(M, \nabla^{M}\right) \wedge \omega-\frac{1}{2} \widetilde{\eta}_{\rho} ; \tau_{c}\right\rangle=\left\langle\operatorname{Ch}\left(\operatorname{Ind}\left(\mathcal{D}_{\rho}^{\Gamma}\right)^{+}\right) ; \tau_{c}\right\rangle .
$$

Using Proposition 1.3 we see that the left hand-side is a homotopy invariant of the pair $(M, \partial M)$. This proves Theorem 0.1 in the even dimensional case. If $M$ is odd dimensional and satisfies the two assumptions $\left(H_{1}\right)$ and $\left(H_{2}\right)$ then we can certainly define the higher twisted eta invariant for the boundary signature operator (see [L2]). The higher signatures are now defined exactly as before

$$
\sigma(M, \partial M ;[c]):=\left\langle\ell \int_{M} L\left(M, \nabla^{M}\right) \wedge \omega-\frac{1}{2} \widetilde{\eta}_{\rho}, \tau_{c}\right\rangle .
$$

In order to show that these numbers are homotopy invariants of the pair $(M, \partial M)$ we follow the usual technique and cross the relavant manifolds by $S^{1}$. Thus let $M$ and $M^{\prime}$ be two homotopy equivalent odd-dimensional manifolds with boundary satisfying $\left(H_{1}\right)$ and $\left(H_{2}\right)$. The manifolds $M \times S^{1}$ and $M^{\prime} \times S^{1}$ are thus even dimensional and still homotopy equivalent. They certainly satisfy assumption $\left(H_{1}\right)$. Let $\mathcal{D}_{M \times S^{1}, \rho}^{\Gamma \times \mathbb{Z}}$ and $\mathcal{D}_{M^{\prime} \times S^{1}, \rho}^{\Gamma \times \mathbb{Z}}$ be the signature operators with values in the flat line bundle $\mathcal{V}_{\rho}$ (see the beginning of this Section). Because of assumption $\left(H_{2}\right)$ on $M, M^{\prime}$ we see easily that $M \times S^{1}$ and $M^{\prime} \times S^{1}$ also satisfy assumption $\left(H_{2}\right)$; in other words these two operators have invertible boundary operators in the Mishenko-Fomenko calculus. They are therefore $C_{r}^{*}(\Gamma \times \mathbb{Z})$-Fredholm with equal higher index classes in $K_{0}\left(C_{r}^{*}(\Gamma \times \mathbb{Z})\right)$. Observe now that by Fourier transform

$$
K_{0}\left(C_{r}^{*}(\Gamma \times \mathbb{Z})\right)=K_{0}\left(C_{r}^{*}(\Gamma) \otimes C^{\infty}(\widehat{\mathbb{Z}})\right) .
$$

Similarly, the higher signature operator $\mathcal{D}_{M \times S^{1}, \rho}^{\Gamma \times \mathbb{Z}}$ can be described in 
terms of Lusztig's family in his $S^{1}$-component, i.e. in terms of the $\widehat{\mathbb{Z}} \equiv S^{1}$-family:

$$
\left(\mathcal{D}_{M} \otimes \operatorname{Id}_{\theta}+\operatorname{Id} \otimes \mathrm{D}_{\theta}\right)_{\theta \in \widehat{\mathbb{Z}}}
$$

It is easy to check that the $S^{1}$-family $\left(\mathcal{D}_{M} \otimes \operatorname{Id}_{\theta}+\operatorname{Id} \otimes \mathrm{D}_{\theta}\right)_{\theta \in \widehat{\mathbb{Z}}}$ is nothing but the Dirac suspension considered in [MP2]. Performing in this setting the computation presented there it is easy to show that the $\widehat{\mathbb{Z}}$-fibre-integral of the Chern character of the index class $\operatorname{Ind}\left(\mathcal{D}_{M \times S^{1}, \rho}^{\Gamma \times \mathbb{Z},+}\right)$ is precisely equal to the noncommutative differential form

$$
\ell \int_{M} L\left(M, \nabla^{M}\right) \wedge \omega-\frac{1}{2} \widetilde{\eta}_{\rho}
$$

appearing in the definition of the odd higher signatures (1.3). Of course the same argument applies to $M^{\prime}$; the equality of the two higher index classes of $M \times S^{1}$ and $M^{\prime} \times S^{1}$ obviously implies the equality of the $\widehat{\mathbb{Z}}$ fibre-integral of their respective Chern characters; this gives the equality

$$
\sigma(M, \partial M ;[c])=\sigma\left(M^{\prime}, \partial M^{\prime} ;[c]\right) \quad \forall[c] \in H^{*}(\Gamma ; \mathbb{C}) .
$$

Summarizing: under assumption $\left(H_{1}\right)$ and $\left(H_{2}\right)$ the numbers $(1.3)$ are homotopy invariants of the pair $(M, \partial M)$, for any $[c] \in H^{*}(\Gamma ; \mathbb{C})$, both for even and odd dimensional manifolds. Our main result is now completely proved.

\section{Examples}

We contruct an explicit example of a manifold $M$ satisfying $\left(H_{1}\right)\left(H_{2}\right)$. Let $p$ be an odd prime integer and consider the 3-dimensional lens space

$$
L\left(p^{2}, p^{2}-1\right) \quad \text { with } \quad \pi_{1}\left(L\left(p^{2}, p^{2}-1\right)\right)=\mathbb{Z}_{p^{2}}
$$

Then [FS] there exists a non-simply connected oriented 4-dimensional manifold $B_{p}$ with boundary

$$
\partial B_{p}=L\left(p^{2}, p^{2}-1\right)
$$

moreover the inclusion $i: L\left(p^{2}, p^{2}-1\right) \rightarrow B_{p}$ induces an epimorphism

$$
\pi_{1}\left(L\left(p^{2}, p^{2}-1\right)\right)=\mathbb{Z}_{p^{2}} \longrightarrow \pi_{1}\left(B_{p}\right)=\mathbb{Z}_{p}
$$

Let $W$ be a closed compact smooth oriented manifold such that $B_{p} \times W$ be even dimensional. Assume $\pi_{1}(W) \equiv \Gamma$ to be either of polynomial TOME $127-1999-\mathrm{N}^{\circ} 2$ 
growth or Gromov hyperbolic. Doing surgery along a submanifold of $\left(B_{p} \times W\right) \backslash \partial\left(B_{p} \times W\right)$ of codimension at least 3 we obtain a compact even dimensional manifold $M$ with boundary equal to $L\left(p^{2}, p^{2}-1\right) \times W$ and with fundamental group equal to $\mathbb{Z}_{p} \times \Gamma$. Clearly there is no reason why such a manifold $M$ should be a product. Let now $\rho: \mathbb{Z}_{p} \rightarrow U(1)$ be a nontrivial representation. Let $\rho_{1}$ be the representation of $\pi_{1}\left(L\left(p^{2}, p^{2}-1\right)\right)$ given by $\rho$ and $(2.1)$. If $\widetilde{E}_{\rho}$ is the flat bundle on $\widetilde{M}^{\Gamma}$ defined by $\rho$, then one verifies that the restriction of $\widetilde{E}_{\rho}$ to the boundary

$$
\partial \widetilde{M^{\Gamma}} \equiv L\left(p^{2}, p^{2}-1\right) \times \widetilde{W}
$$

is equal to the flat bundle induced by the representation $\rho_{1}$. On the other hand it is an easy exercise to show that the twisted signature Laplacian on $L\left(p^{2}, p^{2}-1\right)$ is always invertible; this implies readily that hypothesis $\left(H_{2}\right)$ is satisfied by $M$. Notice that in this case the twisted higher eta invariant has the following simple expression

$$
\widetilde{\eta}_{\rho}=\eta_{\rho}\left(L\left(p^{2}, p^{2}-1\right)\right) \cdot \int_{W} L\left(W, \nabla^{W}\right) \wedge \omega_{W}
$$

REMARK. - More generally the invertibility of the twisted Laplacian on lens spaces can for example be used to imply this invertibility in a number of situations similar to the one presented above. In this context it should be remarked that a formula similar to (2.2) can be proved; notice moreover that the twisted eta invariant of lens spaces can be explicitly computed, see [APS 2].

\section{Remarks on the gap case}

In this section we assume that the group $\Gamma$ is hyperbolic and that $M$ is even-dimensional; we will denote by $\mathcal{B}^{\infty}$ the Connes-Moscovici algebra (see $[\mathrm{CM}]$ ) associated with $\Gamma$. We assume the following on the boundary signature operator:

$$
\left.\exists \delta>0: \quad \operatorname{spec}_{L^{2}} \widetilde{\mathrm{D}_{0}} \cap\right]-\delta, \delta[\subset\{0\}
$$

This gap assumption is satisfied in the following examples.

\section{EXAMPLES}

1) $\Gamma$ is the relative fundamental group of $M$.

2) Let $W$ be a compact quotient of a Hyperbolic space $H^{2 d}$ of dimension $2 d$ and $S^{2 k+1}$ a sphere of dimension $2 k+1$. Let $q$ be a nonnegative 
integer such that the union of $q$ copies of $S^{2 k+1}$ is the boundary of a compact manifold $X$. We assume that $2 k+1+2 d>4$. Let $M$ be a compact manifold obtained from $X \times W$ by doing surgery along a submanifold of $X \times W \backslash \partial X \times W$ of codimension at least 3 , then $M$ will not be a product in general but, using $[\mathrm{L} 4, \mathrm{p} .3]$, one checks easily that the above gap assumption is satisfied for $M$.

We set

$$
\Lambda=C_{r}^{*}(\Gamma) .
$$

Under assumption (3.1), one can prove ( see the Appendix) that the main result (Theorem 14.1, p. 97) of [LP1] can be extended to the hyperbolic case. Thus if $x$ is fixed boundary defining function and $\epsilon>0$ is small enough then, the signature operator $\mathcal{D}^{+}$defines a canonical index class Ind $x^{-\epsilon} \mathcal{D}^{+} x^{\epsilon} \in K_{0}(\Lambda)$, the higher eta invariant $\widetilde{\eta}$ of the boundary Dirac operator is well defined and we have

$$
\begin{aligned}
& \operatorname{Ch}\left(\operatorname{Ind} x^{-\epsilon} \mathcal{D}^{+} x^{\epsilon}\right) \\
& \quad=\int_{M} L(M) \wedge \omega-\frac{1}{2}\left(\widetilde{\eta}+\operatorname{Ch}\left(\operatorname{null}\left(\mathcal{D}_{0}\right)\right)\right) \in \bar{H}_{*}\left(\mathcal{B}^{\infty}\right) .
\end{aligned}
$$

Let $c \in Z^{*}(\Gamma, \mathbb{C})$ be a group cocycle; we can assume that the corresponding cyclic cocycle $\tau_{c}$ of $\mathbb{C}[\Gamma]$ extends as a cyclic cocycle of $\mathcal{B}^{\infty}$. We define the higher signatures of $(M, \partial M)$ as:

$$
\left\langle\int_{M} L(M) \wedge \omega-\frac{1}{2} \widetilde{\eta} ; \tau_{c}\right\rangle .
$$

Now we will follow [Ch1], [Ch2] and add a cone to $M$; this will allow to define a canonical signature index class in $K_{0}(\Lambda)$ which will be an homotopy invariant of the pair $(M, \partial M)$. Lastly, we will state as a conjecture the noncommutative version of the index formula of [ $\mathrm{BiCh}]$ for a family of signature operators on cones; this conjecture would imply the homotopy invariance of the higher signature (3.3) for each $[c] \in H^{*}(\Gamma, \mathbb{C})$.

Now, let us recall that $M$ is an even dimensional compact oriented Riemannian manifold with boundary, we assume that the metric has a product structure near the boundary.

We consider the cone

$$
C(\partial M)=] 0,1] \times \partial M=\{(r, y)\}
$$

endowed with the metric

$$
\mathrm{d} r \otimes \mathrm{d} r+r^{2} g
$$

TOME $127-1999-\mathrm{N}^{\circ} 2$ 
where $g$ is the metric on $\partial M$. We set

$$
M^{\prime}=M \cup C(\partial M)
$$

and denote by $\widetilde{M}^{\prime}$ the associated $\Gamma$-normal cover. We set:

$$
\mathcal{E}^{ \pm}=\left(\widetilde{M^{\prime}} \times_{\Gamma} C_{r}^{*}(\Gamma)\right) \otimes \Lambda^{ \pm} T^{*} M^{\prime}
$$

We denote by $\mathcal{D}^{\prime}\left(\right.$ resp. $\left.\mathcal{D}_{0}\right)$ the induced $C_{r}^{*}(\Gamma)$-signature operator (resp. boundary signature operator) acting on sections of $\mathcal{E}^{+} \oplus \mathcal{E}^{-}\left(\operatorname{resp} \mathcal{E}_{\mid \partial M}^{+}\right)$.

DeFinition 3.1.

$$
\begin{aligned}
\operatorname{Dom} \mathcal{D}^{\prime}=\left\{\alpha \in L^{2}\left(M^{\prime}, \mathcal{E}\right) ; \exists \alpha_{p}\right. & \in C_{\mathrm{comp}}^{\infty}\left(M^{\prime}, \mathcal{E}\right), p \in \mathbb{N} \\
\alpha_{p} & \left.\rightarrow \alpha, \mathcal{D}^{\prime}\left(\alpha_{p}\right) \rightarrow \mathcal{D}^{\prime}(\alpha) \text { in } L^{2}\left(M^{\prime}, \mathcal{E}\right)\right\}
\end{aligned}
$$

Assumption (3.1) allows to adapt easily the proof of Theorem 2.2 of [Ch1] so as to get the next proposition:

Proposition 3.2. - For any $\alpha, \beta$ in $\operatorname{Dom} \mathcal{D}^{\prime}$ :

$$
\left\langle\mathcal{D}^{\prime}(\alpha), \beta\right\rangle=\left\langle\alpha, \mathcal{D}^{\prime}(\beta)\right\rangle
$$

where $\langle$,$\rangle is the \Lambda$-scalar product of the $\Lambda$-Hilbert module $L^{2}\left(M^{\prime}, \mathcal{E}\right)$.

Proposition 3.3. - There exits $\mathrm{e}^{-t \mathcal{D}^{\prime 2}} \in K_{\Lambda}\left(L^{2}\left(M^{\prime}, \mathcal{E}\right)\right)$ for each $t>0$ such that:

$$
\begin{aligned}
& \mathrm{e}^{-t \mathcal{D}^{\prime 2}}\left(L^{2}\left(M^{\prime}, \mathcal{E}\right)\right) \subset \operatorname{Dom} \mathcal{D}^{\prime} ; \quad\left(\frac{\mathrm{d}}{\mathrm{d} t}+\mathcal{D}^{\prime 2}\right) \mathrm{e}^{-t \mathcal{D}^{\prime 2}}=0 \\
& \lim _{t \rightarrow 0^{+}} \mathrm{e}^{-t \mathcal{D}^{\prime 2}}=\mathrm{Id} \text {. }
\end{aligned}
$$

Proof. - The gap assumption (3.1) allows us to adapt easily the construction of the Heat kernel given in [Ch2], for instance the expression of $\mathrm{e}^{-t \Delta}$ given in Example 3.1 of [Ch2] should be replaced in our context by:

$$
\left(r_{1} r_{2}\right)^{\alpha(i)} \frac{1}{2 t} \exp -\left(\frac{r_{1}^{2}+r_{2}^{2}}{4 t}\right) I_{\sqrt{\mathcal{D}_{0}^{2}+\alpha^{2}(i)}}\left(\frac{r_{1} r_{2}}{2 t}\right) .
$$

Proposition 3.4.

1) $\operatorname{Id}+\mathcal{D}^{\prime 2}: \operatorname{Dom} \mathcal{D}^{\prime 2} \rightarrow L^{2}\left(M^{\prime}, \mathcal{E}\right)$ is surjective;

2) $\left(\operatorname{Id}+\mathcal{D}^{\prime 2}\right)^{-1} \in K_{\Lambda}\left(L^{2}\left(M^{\prime}, \mathcal{E}\right)\right)$. 
Proof. - It is a simple consequence of Proposition 3.3 and of the identity:

$$
\left(\mathrm{Id}+\mathcal{D}^{\prime 2}\right) \circ \int_{0}^{+\infty} \mathrm{e}^{-t\left(\mathrm{Id}+\mathcal{D}^{\prime 2}\right)} \mathrm{d} t=\mathrm{Id} .
$$

Proposition 3.5.

1) $\mathcal{D}^{\prime 2}: H_{\Lambda}^{2}\left(M^{\prime}, \mathcal{E}\right) \rightarrow L^{2}\left(M^{\prime}, \mathcal{E}\right)$ is $\Lambda$-Fredholm;

2) $\mathcal{D}^{\prime}: H_{\Lambda}^{1}\left(M^{\prime}, \mathcal{E}\right) \rightarrow L^{2}\left(M^{\prime}, \mathcal{E}\right)$ is $\Lambda$-Fredholm.

Proof. - 2) is a consequence of 1). Let us prove 1).

Let $\phi(t) \in C_{\text {comp }}^{\infty}([0,+\infty[, \mathbb{R})$ be such that $\phi(t)=1$ for $0 \leq t \leq 1$. We then have, thanks to Proposition 3.3:

$$
\mathcal{D}^{\prime 2} \circ \int_{0}^{+\infty} \mathrm{e}^{-t \mathcal{D}^{\prime 2}} \phi(t) \mathrm{d} t=\mathrm{Id}-\int_{0}^{+\infty} \mathrm{e}^{-t \mathcal{D}^{\prime 2}} \phi^{\prime}(t) \mathrm{d} t .
$$

Since $\phi^{\prime}(t)=0$ for $0 \leq t \leq \frac{1}{2}$ and $\mathrm{e}^{-t \mathcal{D}^{\prime 2}}$ is $\Lambda$-compact for $\left.t>0,1\right)$ is proved.

Proposition 3.6. - The index class Ind $\mathcal{D}^{\prime+} \in K_{0}(\Lambda)$ defined by Proposition 3.5 is a homotopy invariant of the oriented pair $(M, \partial M)$.

Proof. - Propositions 3.2, 3.4 and 3.5 allow us to adapt in a straightforward way the proof of [HS] for a closed manifold. We omit the details.

Now, we make the following conjecture which is nothing but the noncommutative version of the Index theorem of [BiCh] (see p. 70) for a family of signature operators on cones such that the kernels of the boundary operators have constant rank.

ConJeCture 3.7. - Under assumption (3.1) we have:

$$
\mathrm{Ch} \text { Ind } \mathcal{D}^{\prime+}=\int_{M} L(M) \wedge \omega-\frac{1}{2} \tilde{\eta} \in \bar{H}_{*}\left(\mathcal{B}^{\infty}\right)
$$

where the higher eta invariant $\widetilde{\eta}$ is exactly the one in (3.2).

REMARK. - There are two possible routes to this conjecture. One is to prove that

$$
\operatorname{Ch}\left(\operatorname{Ind}\left(\mathcal{D}^{\prime+}\right)\right)=\operatorname{Ch}\left(\operatorname{Ind} x^{-\varepsilon} \mathcal{D}^{+} x^{\varepsilon}\right)+\frac{1}{2} \operatorname{Ch}\left(\operatorname{null}\left(\mathcal{D}_{0}\right)\right) .
$$

When $\Gamma$ is trivial the latter result is true and proved by combining arguments of [Me, chap.6] and of [Ch1], [Ch2]. Notice, however, that

TOME $127-1999-\mathrm{N}^{\circ} 2$ 
these arguments use Hodge theory on the whole manifold in an essential way; extending these Hodge-theoretic arguments to the noncommutative case does not seem straighforward. A second approach would be to prove formula (3.4) directly, with no reference to the $b$-calculus.

Under assumption (3.1), Conjecture 3.7 and Proposition 3.6 imply that (3.3) is an homotopy invariant of the oriented pair $(M, \partial M)$ for each $[c] \in H^{*}(\Gamma, \mathbb{C})$.

\section{Appendix: The higher APS index formula for finitely presented groups}

In this section we will make use of a recent paper of Lott [L3] in order to extend the main result of [LP1] (when the $L^{2}$-spectrum of the lifted boundary operator has a gap at zero) to groups which are only finitely presented. Notice however that all results concerning higher signatures require the extendibility property of the cyclic cocycle $\tau_{c} \in H C^{*}(\mathbb{C} \Gamma)$ associated to $[c] \in H^{*}(\Gamma, \mathbb{C})$ to a cyclic cocycle in $H C^{*}\left(\mathcal{B}^{\infty}\right)$. It is at this point that crucial use is made of the assumption $\Gamma$ Gromov hyperbolic or virtually nilpotent.

As usual we assume some familiarity with the notations and results of [L1], [L2], [LP1].

Thus $M$ is an even dimensional compact manifold with boundary, $E$ is a $\mathbb{Z}_{2}$-graded hermitian vector bundle on $M, \not D$ is an odd self-adjoint Dirac operator acting on the sections of $E$. We consider $\Gamma$, a finitely presented discrete group and $\pi: \widetilde{M} \rightarrow M$, a Galois $\Gamma$-cover of $M$. We let $\widetilde{D}$ be the lifted $\Gamma$-invariant Dirac operator on $\widetilde{M}$ acting on the sections of $\widetilde{E}=\pi^{*}(E)$; we denote by $\not_{0}, \widetilde{D}_{0}$ the Dirac operators induced on the boundary. Finally we introduce the $C^{*}$-algebra $\Lambda \equiv C_{r}^{*}(\Gamma)$ and the flat $\Lambda$-bundle on $M: \mathcal{V}=\widetilde{M} \times_{\Gamma} \Lambda$. We let $\mathcal{E}=\mathcal{V} \otimes E$. The operator $\not D$ induces a $\Lambda$-differential operator $\not \supset$ acting on the sections of $\mathcal{E}$ with boundary operator $\not_{0}$. The boundary operators $\not_{0}, \widetilde{D}_{0}$ and $\not_{0}$ are self-adjoint with respect to the appropriate scalar products. We refer to [LP1] for more on these geometric preliminaries and also for the material concerning Lott's connection $\nabla$ (see respectively Sect. 4 and Sect. 2). As a last piece of background material we introduce the algebra

$$
\mathcal{B}^{\infty}=\left\{T \in C_{r}^{*}(\Gamma) ; \forall k \in \mathbb{N}, \delta^{k}(T) \text { is bounded on } \ell^{2}(\Gamma)\right\}
$$

where $\delta(T)=[D, T]$ and $D$ is the unbounded operator on $\ell^{2}(\Gamma)$ associated with a word metric $L$

$$
D\left(\varepsilon_{h}\right)=L(h) \varepsilon_{h}
$$


for any $h \in \Gamma\left(\left(\varepsilon_{h}\right)\right.$ denotes the standard orthonormal basis of $\left.\ell^{2}(\Gamma)\right)$. It is well known that $\mathcal{B}^{\infty}$ is dense in $C_{r}^{*}(\Gamma)$ and closed under holomorphic functional calculus.

The goal of this Appendix is to prove the following theorem, extending to arbitrary finitely presented discrete groups $\Gamma$ the results of [LP1]:

ThEOREM 4.1. - Assume that there exits $\delta>0$ such that

$$
\operatorname{spec}_{L^{2}} \widetilde{D D}_{0} \cap[-\delta, \delta] \subset\{0\}
$$

Then

1) The higher eta invariant $\widetilde{\eta} \equiv \widetilde{\eta}\left(\not_{0}\right)$ of Lott [L2] is well defined as an element of $\overline{\widehat{\Omega}}\left(\mathcal{B}^{\infty}\right)$.

2) For $\epsilon>0$ small enough, $x^{-\epsilon} \not \not^{+} x^{\epsilon}$ is $\mathcal{B}^{\infty}$-Fredholm and thus defines an index class $\operatorname{Ind}\left(\not{D}^{+}\right) \in K_{0}\left(\mathcal{B}^{\infty}\right)$.

3) The null space null $\not_{0}$ is a finitely generated $\mathcal{B}^{\infty}$-projective module. For the Chern character of $\operatorname{Ind}\left(\not^{+}\right)$, in the noncommutative topological de Rham homology of $\mathcal{B}^{\infty}$, the following formula holds:

$$
\operatorname{Ch}\left(\operatorname{Ind}\left(\not \not^{+}\right)\right)=\int_{M} \widehat{A} \wedge \mathrm{Ch}^{\prime}(E) \wedge \omega-\frac{1}{2}\left(\widetilde{\eta}+\operatorname{Ch} \text { null } \not \not_{0}\right)
$$

The proof will in fact establish the convergence of Lott's higher eta invariant for any closed manifold, not necessarily the boundary of an even dimensional manifold. This convergence is implicitly proved in [L3], where a new technique for studying the large time behaviour of the superconnection heat kernel associated to $\not_{0}$ is introduced.

We now prove [1]. As in [L3] we consider $\mathcal{B}^{\infty}$ as the projective limit of the sequence of Banach algebras $\left(B_{j}\right)_{j \geq 0}$ with norms $|\cdot|_{j}$ :

$$
\cdots \rightarrow B_{j} \longrightarrow B_{j-1} \longrightarrow \cdots \longrightarrow B_{0}
$$

with $B_{0}=\Lambda$. The definition of $B_{j}$ is the obvious one, namely:

$$
B_{j}=\left\{T \in C_{r}^{*}(\Gamma) ; \forall k \in\{0,1, \ldots, j\}, \delta^{k}(T) \text { is bounded on } \ell^{2}(\Gamma)\right\}
$$

and

$$
|T|_{j}=2^{j} \sup _{0 \leq k \leq j}\left\|\delta^{k}(T)\right\|_{\ell^{2}(\Gamma) \rightarrow \ell^{2}(\Gamma)}
$$

TOME $127-1999-\mathrm{N}^{\circ} 2$ 
As pointed out in [L3] the norms $|\cdot|_{j}$ on $B_{j}$ induce norms $\|\cdot\|_{j}$ on the space of differential forms of degree $k$ defined in [L1]: $\widehat{\Omega}_{k}\left(\mathcal{B}^{\infty}\right)$ and $\bar{\Omega}_{k}\left(\mathcal{B}^{\infty}\right)$. We denote by

$$
\widehat{\Omega}_{k}\left(B_{j}\right), \quad \overline{\widehat{\Omega}}_{k}\left(B_{j}\right)
$$

the corresponding completions.

For each pair of non-negative integers $j, k$ we introduce the following Banach space

$$
E(j, k) \equiv C^{0}\left(\partial M ;\left(\partial \widetilde{M} \times_{\Gamma} B_{j}\right) \otimes_{\mathbb{C}} E\right) \otimes_{B_{j}} \widehat{\Omega}_{k}\left(B_{j}\right) .
$$

By assumption there exists a $\delta>0$ such that $\left.\operatorname{spec}\left(\not_{0}\right) \cap\right]-\delta, \delta[\subset\{0\}$. Let $\gamma$ be the following contour of the half-plane $x>0: \gamma$ is the union of the two half-lines with slopes \pm 1 and with origin in the point $\left(\frac{1}{2} \delta, 0\right)$. We orient $\gamma$ clockwise.

For each $z \in \gamma$ the operator $\left(z \operatorname{Id}-\not D_{0}^{2}\right)$ is invertible in the $\Lambda$ pseudodifferential calculus. Thus, according to [L3, Prop. 19], it is invertible in the corresponding $B_{j}$ and $\mathcal{B}^{\infty}$-calculi. Moreover, one checks easily that the orthogonal projection $P_{0}$ onto null $\not_{0}$ (given by a contour integral) is a $\mathcal{B}^{\infty}$-smoothing operator. The operators

$$
\mathrm{e}^{-t \not_{0}^{2}}=\frac{i}{2 \pi} \int_{\gamma} \frac{\mathrm{e}^{-t z}}{z \mathrm{Id}-\not{D}_{0}^{2}} \mathrm{~d} z+P_{0}
$$

are therefore well defined and for $t>0$ form a semigroup acting on the Banach space $E(j, k)$. Thus, following again [L3], we have the crucial estimate

$$
\left\|\mathrm{e}^{-t \not_{0}^{2}}-P_{0}\right\|_{E(j, k) \rightarrow E(j, k)} \leq C_{j, k} e^{-t \delta / 4}, \quad \forall t \geq 1 .
$$

Now let us fix $\epsilon>0$. The three following facts are easily seen to be true:

(i) $\not_{0} e^{-\epsilon \not{D}_{0}^{2}}: E(j, k) \rightarrow E(j, k)$ is bounded.

(ii) The commutator

$$
\left[\Upsilon \nabla \not \not_{0}+\not \not_{0} \Upsilon \nabla, \not \not_{0}\right]: E(j, k) \rightarrow E(j, k+1)
$$

is bounded. Here $\Upsilon$ is the obvious grading on $\mathcal{E}_{0} \oplus \mathcal{E}_{0}, \mathcal{E}_{0}=\mathcal{E}_{\partial M}^{+}$, introduced in [LP1]. 
(iii) The commutator

$$
\left[\nabla^{2}, \not_{0}\right]: E(j, k) \rightarrow E(j, k+2)
$$

is bounded.

Now we can prove the convergence of the integral

$$
\frac{2}{\sqrt{\pi}} \int_{0}^{+\infty} \operatorname{STR}_{\mathrm{Cl}(1)}\left(\sigma \not \not_{0} \exp \left(-\left(\Upsilon \nabla+s \sigma \not{D}_{0}\right)^{2}\right)\right) \mathrm{d} s
$$

defining the higher eta invariant. Recall that in this definition $\sigma$ is the involution on $\mathcal{E}_{0} \oplus \mathcal{E}_{0}$, sending $u \oplus v$ to $v \oplus u$. As observed in [L2] we have only to prove the convergence when $s \rightarrow+\infty$. Let us fix $E(j, k)$; we follow closely the Berline-Getzler-Vergne argument used in section 2 of [LP1] but we work in the $B_{j}$-calculus on the base (instead of the covering), thus we consider $\left(\Upsilon \nabla+s \sigma \not \not_{0}\right)^{2}$ as a perturbation of $\left(s \not \not_{0}\right)^{2}$ viewed as a $B_{j}$-operator. The estimate (4.2) and the three facts above can now be used (as in Section 2 of [LP1]) in order to prove the convergence of the integral (4.3) for the norm $\|\cdot\|_{j}$. Since this is true for any $j, 1$ ) is proved.

In order to prove 2) we remark that in the virtually nilpotent case treated in [LP1] this property is the consequence of the $b-\mathcal{B}^{\infty}$-MishenkoFomenko decomposition theorem. The latter is proved following the ideas in $[\mathrm{MF}]$ and the crucial fact that if a $\mathcal{B}^{\infty}$-pseudodifferential operator is invertible in the $\Lambda$-calculus then it is also invertible in the $\mathcal{B}^{\infty}$-calculus. Since this last property has now been established by Lott [L3] for the algebra $\mathcal{B}^{\infty}$ considered in this section, we see that our arguments in [LP1] apply verbatim to the present situation (see [LP1, Thm 12.7]).

We come finally to the proof of the higher Atiyah-Patodi-Singer formula (4.1). Recall that the proof given in [LP1] is ultimately a consequence of the $b$-heat calculus on the covering and of the $b-\mathcal{B}^{\infty}$-Mishenko-Fomenko calculus and, in particular, of an extension of Melrose' formula for the $b$ trace of a commutator. The $b$-heat calculus on the covering $\widetilde{M}$ is replaced now by a $b-\mathcal{B}^{\infty}$-heat calculus on $M$ to be developed with no difficulties along the lines in [Me]. The part involving the $b-\mathcal{B}^{\infty}$-Mishenko-Fomenko calculus remains unchanged. We follow closely the structure of the proof of [LP1, section 14], so in a first step we assume that $\widetilde{\not D}_{0}$ is invertible. Recall now that by making a series of deformations (inspired by the work of Bismut, see [B], [L1]) one can prove that for $u>0$

$$
\operatorname{Ch}\left(\operatorname{Ind}\left(\not \not^{+}\right)\right)=\int_{M} \widehat{A} \wedge \mathrm{Ch}^{\prime}(E) \wedge \omega-\frac{1}{2} \int_{0}^{u} \widetilde{\eta}(s) \mathrm{d} s+B(u)
$$

TOME $127-1999-\mathrm{N}^{\circ} 2$ 
with $B(u)$ explicit boundary terms and $\widetilde{\eta}(s)$ the integrand of (4.3). One key step in the proof of (4.1) given in [LP1] is to show that as $u \rightarrow+\infty$ the term $B(u)$ converges to zero. It is here that the assumption $\Gamma$ virtually nilpotent is used in a crucial way. We are now going to show that, thanks to the recent results of [L3], these steps can be generalized to the general case. To this end we introduce for each pair of non-negative integers, the Banach space

$$
F(j, k) \equiv C^{1 / 2}\left(\partial M ;\left(\partial \widetilde{M} \times_{\Gamma} B_{j}\right) \otimes_{\mathbb{C}} E\right) \otimes_{B_{j}} \widehat{\Omega}_{k}\left(B_{j}\right) .
$$

The semigroup $\exp \left(-t \not_{0}^{2}\right)$ acting on $F(j, k)$ is again well defined and the estimate (estimate) (with $P_{0}=0$ ) holds with $F(j, k)$ in place of $E(j, k)$. Now we observe that for each real $\lambda$ the operators

$$
\left(\lambda i+\not \not_{0}\right)^{-1} \circ \nabla^{2} \circ\left(\lambda i+\not \not_{0}\right), \quad\left(\lambda i+\not{D}_{0}\right)^{-1} \circ \Upsilon \nabla \circ\left(\lambda i+\not \not_{0}\right) \circ \Upsilon \nabla
$$

are bounded operators from $F(j, k)$ to $F(j, k+2)$. Similarly the operator

$$
\left(\lambda i+\not_{0}\right)^{-1} \circ\left(\Upsilon \nabla \not_{0}+\not_{0} \Upsilon \nabla\right) \circ\left(\lambda i+\not_{0}\right)
$$

is bounded as an operator from $F(j, k) \rightarrow F(j, k+1)$. Here we have used the well known fact that 0 -th order pseudodifferential operators are bounded on the Hölder space $C^{1 / 2}$; notice that this is not the case for the space of continuous functions. By applying Duhamel's formula as above, the analogue of the estimate (estimate) and the two previous remarks one proves, as in [LP1], that the extra boundary terms $B(u)$ converge to 0 as $u \rightarrow+\infty$. Now when $\not_{0}$ is not invertible, one proceeds as in the end of section 14 of [LP1], one considers the $x^{-\epsilon} \not D_{x} \epsilon$, for $\epsilon$ small enough its boundary operator is invertible, then one uses a straightforward generalization of Theorem 3.2 of [LP1] in order to get $-\frac{1}{2}\left(\widetilde{\eta}+\mathrm{Ch}\right.$ null $\left.\not \not_{o}\right)$ as a boundary correction term. The theorem is proved.

REMARK 4.2. - The applications of Theorem 4.1 to positive scalar questions given in [LP1, Section 15] can now be extended to an arbitrary finitely presented group.

\section{BIBLIOGRAPHIE}

[APS1] Atiyah (M.F.), Patodi (V.), Singer (I.). - Spectral Asymmetry and Riemannian Geometry, I, Math. Proc. Cambridge Phil. Soc., t. 77, p. 43,1975 . 
[APS2] Atryah (M.F.), Patodi (V.), , Singer (I.). - Spectral Asymmetry and Riemannian Geometry, I, Math. Proc. Cambridge Phil. Soc., t. 78, p. 405, 1976.

[AS] Aтryah (M.F.), Singer (I.). - The index of elliptic operators, III, Ann. Math., t. 87, 1968, p. 546.

[BiCh] Bismut (J.-M.), Cheeger (J.). - Remarks on The Index Theorem for Families of Dirac Operators on Manifolds with Boundary. Differential Geometricy, B. Lawson and K. Teneblat (eds), Longman Scientific, 1992.

[Ch1] Cheeger (J.). - On the Hodge Theory of Riemannian Pseudomanifolds, Proc. Symposia Pure Mathematics, vol. 36, p. 91, 1980.

[Ch2] Cheeger (J.). - Spectral Geometry of Singular Riemann Spaces, J. Diff. Geom., t. 18, 1983 , p. 575.

[Co] Connes (A.). - Noncommutative geometry. - Academic Press, 1994.

[CM] Connes (A.), Moscovici (H.). - Cyclic Cohomology, the Novikov Conjecture and Hyperbolic Groups, Topology, t. 29, 199o, p. 345.

[FS] Fintushel (R.), Stern (R.). - Rational blowdowns of smooth 4manifolds, J. Diff. Geom., t. 46, 1997, p. 181.

[FRR] Ferry (S.), Ranicki (A.), Rosenberg (J.). - A history and survey of the Novikov conjecture, in Novikov Conjetures, Index Theorems and Rigidity, vol. 1, London Math. Soc., Lecture Notes Series, t. 226, 1995 .

[Ka] Karoubi (M.). - Homologie Cyclique et K-Théorie, Astérisque, t. 149, 1987 .

[HS] Hilsum (M.), Skandalis (G.). - Invariance par homotopie de la signature à coefficient dans un fibré presque plat, J. reine angew. Math., t. 423, 1992, p. 73.

[LP1] Leichtnam (E.), Piazza (P.). - The b-pseudodifferential calculus on Galois coverings and a higher Atiyah-Patodi-Singer index theorem, Mémoires Soc. Math. France, t. 68, 1997.

[LP2] Leichtnam (E.), Piazza (P.). - Spectral sections and higher AtiyahPatodi-Singer index theory on Galois coverings, GAFA, t. 8, 1996, p. 17.

[LP 3] Leichtnam (E.), Piazza (P.). - Higher eta invariants and the Novikov conjecture on manifolds with boundary, C. R. Acad. Sciences, t. 327 serie I, 1998, p. 497-502.

[L1] Lotт (J.). - Superconnections and higher index theory, GAFA, t. 2, 1992, p. 421.

[L2] Lotт (J.). - Higher eta invariants, K-Theory, t. 6, 1992, p. 191. 
[L3] Lott (J.). - Diffeomorphisms, Analytic Torsion and Non Commutative Geometry, Memoires AMS (in press), circulating as Preprint Max Planck Institute, Bonn, 1996.

[L4] LotT (J.). - The Zero-in-the-Spectrum Question, Enseign. Math., t. 42, 1996, p. 341-376.

[Lu] Lusztig (G.). - Novikov's Higher Signature and Families of Elliptic Operators, J. Differential. Geom., t. 7, 1971, p. 229.

[Me] Melrose (R.).- The Atiyah-Patodi-Singer Index Theorem.-A. and K. Peters, 1993 .

[MP1] Melrose (R.), Piazza (P.). - Families of Dirac operators, boundaries and the b-calculus, J. Differential. Geom., t. 46, n ${ }^{\circ}$ 1, 1997, p. 99.

[MP2] Melrose (R.), Piazza (P.). - An index theorem for families of Dirac operators on odd dimensional manifolds with boundary, J. Differential. Geom., t. 46, $\mathrm{n}^{\circ}$ 2, 1997, p. 287.

[Pi] Piazza (P.). - Dirac operators, heat kernels and microlocal analysis, Part I, Rend. Circolo Mat. Palermo, t. 49, 1997, p. 187. 